\title{
Early results of a real-world series with two transapical transcatheter mitral valve replacement devices
}

\author{
S. Ludwig ${ }^{1,2}$ (]) D. Kalbacher ${ }^{1,2} \cdot$ N. Schofer ${ }^{1} \cdot$ A. Schäfer ${ }^{3} \cdot$ B. Koell ${ }^{1} \cdot$ M. Seiffert ${ }^{1,2} \cdot$ J. Schirmer $^{3} \cdot$ U. Schäfer ${ }^{4}$. \\ D. Westermann ${ }^{1,2} \cdot$ H. Reichenspurner ${ }^{2,3} \cdot$ S. Blankenberg ${ }^{1,2} \cdot$ E. Lubos $^{1} \cdot$ L. Conradi ${ }^{2,3}$
}

Received: 10 August 2020 / Accepted: 2 October 2020 / Published online: 19 October 2020

(c) The Author(s) 2020

\begin{abstract}
Aims Transcatheter mitral valve replacement (TMVR) with dedicated devices promises to fill the treatment gap between open-heart surgery and edge-to-edge repair for patients with severe mitral regurgitation (MR). We herein present a singlecentre experience of a TMVR series with two transapical devices.

Methods and results A total of 11 patients were treated with the Tendyne ${ }^{\mathrm{TM}}(N=7)$ or the Tiara ${ }^{\mathrm{TM}}$ TMVR systems $(N=4)$ from 2016 to 2020 either as compassionate-use procedures or as commercial implants. Clinical and echocardiographic data were collected at baseline, discharge and follow-up and are presented in accordance with the Mitral Valve Academic Research Consortium (MVARC) definitions.

The study cohort [age 77 years $(73,84) ; 27.3 \%$ male] presented with primary $(N=4)$, secondary $(N=5)$ or mixed $(N=2)$ MR etiology. Patients were symptomatic (all NYHA III/IV) and at high surgical risk [logEuroSCORE II 8.1\% (4.0, 17.4)]. Rates of impaired RV function (72.7\%), severe pulmonary hypertension (27.3\%), moderate or severe tricuspid regurgitation $(63.6 \%)$ and prior aortic valve replacement $(63.6 \%)$ were high. Severe mitral annulus calcification was present in two patients. Technical success was achieved in all patients. In 90.9\% $(N=10)$ MR was completely eliminated (i.e. no or trace MR). Procedural and 30-day mortality were $0.0 \%$. At follow-up NYHA class was I/II in the majority of patients. Overall mortality after 3 and 6 months was $10.0 \%$ and $22.2 \%$.

Conclusions TMVR was performed successfully in these selected patients with complete elimination of MR in the majority of patients. Short-term mortality was low and most patients experienced persisting functional improvement.
\end{abstract}

Electronic supplementary material The online version of this article (https://doi.org/10.1007/s00392-020-01757-z) contains supplementary material, which is available to authorized users.

Extended author information available on the last page of the article 


\section{Graphic abstract}

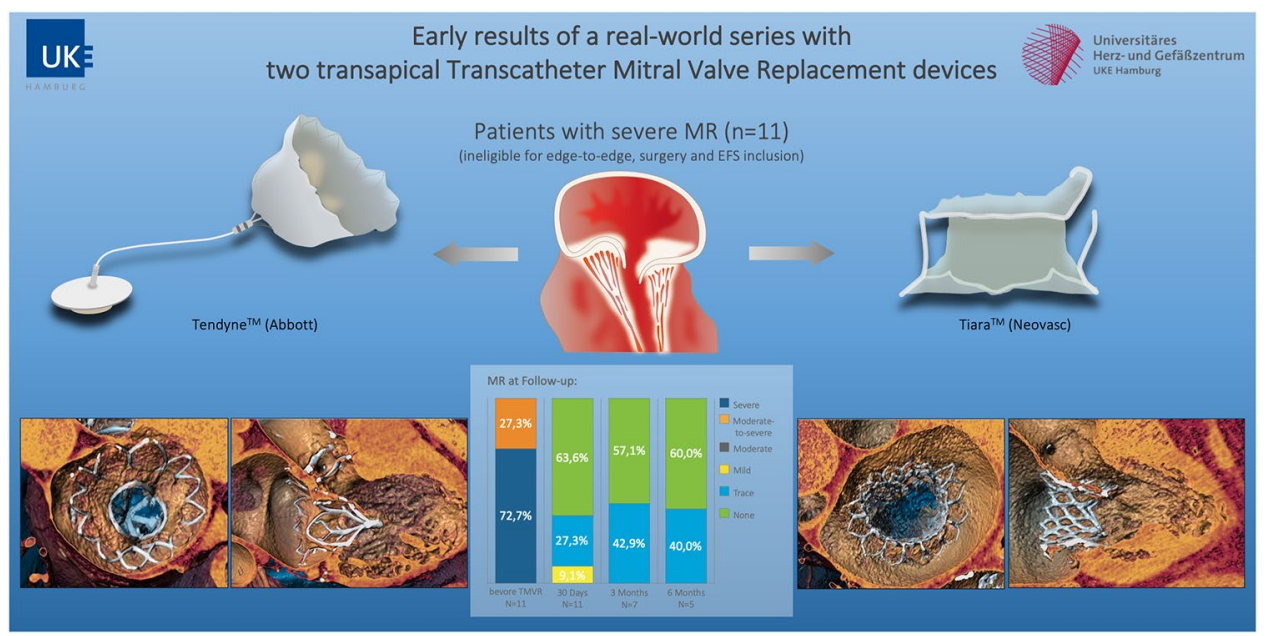

Keywords Transcatheter mitral valve replacement · Mitral regurgitation $\cdot$ Compassionate use

\section{Introduction}

In recent years, transcatheter mitral valve replacement (TMVR) has evolved to an alternative treatment option for high-risk patients suffering from severe mitral regurgitation (MR) $[1,2]$. Several ongoing early feasibility trials have shown favourable procedural and short-term outcomes with different dedicated TMVR systems in selected patient populations [3, 4]. However, a large portion of severe MR patients has so far been precluded from TMVR due to strict anatomical or clinical study exclusion criteria $[5,6]$. If these patients rejected for conventional endovascular MR therapy are continuously treated medically following TMVR screening failure, notably high mortality rates after screening initiation have been reported [7-9].

While some studies suggest that TMVR is safe and feasible in patients with sensitive anatomical conditions, such as severe mitral annular calcification (MAC) or prior aortic valve replacement $[10,11]$, real-world data of patients treated with TMVR outside of feasibility trials are scarce. With this study, we present a single-centre experience of 11 patients with severe MR treated with two different dedicated transapical TMVR devices, either as part of a compassionate-use (CU) program or as early commercial (CE) implants.

\section{Methods}

\section{Study population}

From 2016 to 2020, a total of 115 patients with moderateto-severe or severe MR were screened for TMVR at the
University Heart and Vascular Center Hamburg, Hamburg, Germany. Of these, 35 patients underwent TMVR with four dedicated transapical or transseptal devices [Tendyne ${ }^{\mathrm{TM}}$ (Abbott Vascular, Abbott Park, IL, USA), Tiara ${ }^{\mathrm{TM}}$ (Neovasc Inc., New Brighton, MN, USA), CardiAQ ${ }^{\mathrm{TM}}$ (Edwards Lifesciences, Irvine, CA, USA), HighLife Valve ${ }^{\mathrm{TM}}$ (HighLife Medical, Paris, France)]. Twenty-two patients were treated as part of early feasibility trials. Ten patients received TMVR as part of CU programs (Tendyne ${ }^{\mathrm{TM}}$, Tiara $^{\mathrm{TM}}$, Car$\mathrm{di}^{\mathrm{A}} \mathrm{Q}^{\mathrm{TM}}$ ) and 3 patients were treated as CE implants (only Tendyne $^{\mathrm{TM}}$ ). For the present study, we excluded patients included in early feasibility trials and those treated with devices no longer available (i.e. CardiAQ ${ }^{\mathrm{TM}}$ ). Hence, we report a series of 11 patients treated with either the Tendyne $^{\mathrm{TM}}$ TMVR system $(N=7)$ or the Tiara ${ }^{\mathrm{TM}}$ TMVR system $(N=4)$. Both TMVR devices were implanted via transapical access.

\section{Data acquisition and follow-up}

Baseline, procedural and discharge information as well as survival data were obtained from in-house information as part of clinical routine and presented in accordance with the mitral valve academic research consortium (MVARC) definitions. All patients provided written informed consent for device implantation and data acquisition. The study was conducted in accordance with the Declaration of Helsinki.

\section{Pre-procedural screening}

All patients with severe MR were primarily screened for endovascular edge-to-edge repair. Severe leaflet 
calcification, small valve orifice, high transvalvular gradients and large coaptation gap represent the most frequent causes for the decision against endovascular edge-to-edge repair. The screening process for the assessment of TMVR eligibility comprised multislice computed tomography (MSCT) and echocardiography (transthoracic and transesophageal). Common reasons for anatomical TMVR ineligibility were small left ventricle (LV) dimensions, high risk of left ventricular outflow tract (LVOT) obstruction, severe circumferential MAC and annular size. A detailed description of the screening process including the decision of an interdisciplinary heart team has been given elsewhere [5]. Reasons for the decision against endovascular edge-to-edge repair for all patients and early feasibility study exclusion criteria are given in Supplementary Table 1.

\section{Echocardiography and MSCT}

Transthoracic and transesophageal echocardiography were performed in all patients to assess MR severity and etiology. Evaluation and grading of MR were performed in line with the 2017 ESC/EACTS guidelines for the management of valvular heart disease and documented according to a standardized protocol [12]. Full cardiac cycle MSCT was performed in every patient. Dimensions of the mitral valve annulus and the LV were assessed at 30\% (end-systole) and $75 \%$ (mid-to-end diastole) of the cardiac cycle with a dedicated software (3mensio Structural Heart V10.0, Pie Medical Imaging, Maastricht, Netherlands). The mitral valve annulus was measured according to a D-shaped annulus concept, as described before [13].

\section{Tendyne $^{T M}$ TMVR system}

The Tendyne ${ }^{\mathrm{TM}}$ TMVR system is a self-expanding device with transapical delivery approach. It consists of a nitinolbased, dual-frame structure with an outer frame contoured to the shape of the mitral annulus and a circular inner frame holding the tri-leaflet bovine pericardial valve. The Tendyne $^{\mathrm{TM}}$ device is characterized by a high-molecular-weight polyethylene tether that connects the prosthesis to the apex where it is attached to an epicardial pad. There are multiple valve size configurations ranging from AP diameter $29-41 \mathrm{~mm}$ and CC diameter 34-53 mm [1, 14, 15].

\section{Tiara $^{\mathrm{TM}}$ TMVR system}

The Tiara ${ }^{\mathrm{TM}}$ TMVR device is a D-shaped, nitinol alloybased, self-expanding device consisting of a frame and a tri-leaflet bovine pericardial valve. It is implanted via transapical access. Three ventricular anchors (two anterior, one posterior) secure fixation of the valve onto the fibrous trigons and the posterior part of the annulus. A circular atrial skirt helps to seal and stabilize the device from the atrial side of the mitral annulus and the aortomitral continuity. Currently, there are two Tiara ${ }^{\mathrm{TM}}$ device sizes: $35 \mathrm{~mm}$ (AP 26.5-30.0 mm, CC 31.0-35.0 mm, annulus area 6.5-9.0 $\mathrm{cm}^{2}$ ) and $40 \mathrm{~mm}$ (AP $30.0-34.0 \mathrm{~mm}$, CC $35.0-40.0 \mathrm{~mm}$, annulus area $\left.9.0-12.0 \mathrm{~cm}^{2}\right)[1,16,17]$.

\section{Statistical analysis}

Continuous variables were shown as median [interquartile range (IQR)] and binary variables were shown as absolute numbers and percentages. Echocardiographic parameters before and after TMVR were compared using a paired sample $t$ test.

\section{Results}

\section{Clinical baseline characteristics}

Detailed clinical baseline characteristics of the study population $(n=11)$ are given in Table 1 . The patients (77 years [73, 84], male $N=3$ ) presented with high rates

Table 1 Clinical baseline characteristics of the study population $(n=11)$

\begin{tabular}{ll}
\hline Clinical baseline characteristics & $n=11$ \\
\hline Age (years) & $77(73,84)$ \\
Female gender & $8(72.7)$ \\
Body mass index $\left(\mathrm{kg} / \mathrm{m}^{2}\right)$ & $25.5(21.6,29.5)$ \\
logEuroSCORE II $(\%)$ & $8.1(4.0,17.4)$ \\
STS PROM (\%) & $4.1(2.6,7.3)$ \\
Diabetes & $1(9.1)$ \\
COPD & $2(18.2)$ \\
Impaired renal function $(\mathrm{GFR}<50 \mathrm{ml} / \mathrm{min})$ & $10(90.9)$ \\
Chronic haemodialysis & $2(18.2)$ \\
Immunosuppression & $5(45.5)$ \\
Atrial fibrillation & $9(81.8)$ \\
Prior myocardial infarction & $2(18.2)$ \\
Prior cardiac surgery & $4(36.4)$ \\
Prior CABG & $2(18.2)$ \\
Prior aortic valve replacement (TAVI or SAVR) & $7(63.6)$ \\
Prior TAVI & $5(45.5)$ \\
Prior SAVR & $2(18.2)$ \\
Heart failure hospitalization (last year) & $10(90.9)$ \\
NYHA stage III & $10(90.9)$ \\
NYHA stage IV & $1(9.1)$ \\
\hline
\end{tabular}

$C A B G$ coronary artery bypass graft, $C O P D$ chronic obstructive pulmonary disease, GFR glomerular filtration rate, NYHA New York Heart Association, SAVR surgical aortic valve replacement, STS PROM Society of Thoracic Surgeons Predicted Risk of Mortality, TAVI transcatheter aortic valve replacement 
of impaired renal function ( $N=10,90.9 \%)$, immunosuppression $(N=5,45.5 \%)$ and concomitant atrial fibrillation $(N=9,81.8 \%)$. Prior cardiac surgery had been performed in $36.4 \%(N=4)$, of whom $18.2 \%(N=2)$ were treated by coronary artery bypass grafting (CABG). $63.6 \%(N=7)$ of the study population had previously undergone aortic valve replacement $(N=5,45.5 \%$ interventional; $N=2$, $18.2 \%$ surgical). All patients were symptomatic according to New York Heart Association (NYHA) classes III $(N=10,90.9 \%)$ or IV $(N=1,9.1 \%)$. The above conditions translated into elevated surgical risk as assessed by $\operatorname{logEuroSCORE~II~} 8.1 \%(4.0,17.4)$ and STS PROM $4.1 \%$ $(2.6,7.3)$.

\section{Echocardiographic and MSCT parameters}

Echocardiographic and MSCT parameters are presented in detail in Table 2. Etiology of MR was functional (FMR), degenerative (DMR) or mixed FMR/DMR in $45.5 \%(N=5)$, $36.4 \%(N=4)$ and $18.2 \%(N=2)$, respectively. Severity of MR was moderate-to-severe $(3+)$ in $27.3 \%(N=3)$ and severe $(4+)$ in $72.7 \%(N=4)$ of the patients. Left ventricular ejection fraction (LVEF) was $45.0 \%(35.0,53.5)$, while in $18.2 \%(N=2)$ of the patients LVEF was severely reduced. Reduced stroke volume index $\left(\mathrm{SVI}<35 \mathrm{ml} / \mathrm{m}^{2}\right)$ at baseline was found in more than half of the study population $(N=6$, $54.5 \%)$. Impaired right ventricular (RV) function was present in $72.7 \%(N=8)$ of all patients with a high median systolic

Table 2 Echocardiographic and computed tomography parameters

\begin{tabular}{|c|c|}
\hline Echocardiographic parameters & $n=11$ \\
\hline MR $3+(\%)$ & $3(27.3)$ \\
\hline MR $4+(\%)$ & $8(72.7)$ \\
\hline FMR $(\%)$ & $5(45.5)$ \\
\hline $\operatorname{DMR}(\%)$ & $4(36.4)$ \\
\hline Mixed FMR/DMR (\%) & $2(18.2)$ \\
\hline Pmean $(\mathrm{mmHg})$ & $4.0(3.0,4.8)$ \\
\hline EROA $\left(\mathrm{cm}^{2}\right)$ & $0.39(0.31,0.52)$ \\
\hline LVEDV (mL) & $114.6(92.1,163.8)$ \\
\hline $\operatorname{LVEF}(\%)$ & $45.0(35.0,53.5)$ \\
\hline Severely reduced $\mathrm{LVEF} \leq 30 \%$ & $2(18.2)$ \\
\hline $\mathrm{SVI}\left(\mathrm{ml} / \mathrm{m}^{2}\right)$ & $31.2(21.2,38.6)$ \\
\hline Reduced $\mathrm{SVI}<35 \mathrm{ml} / \mathrm{m}^{2}$ & $6(54.5)$ \\
\hline TAPSE (mm) & $16.0(14.5,17.0)$ \\
\hline Impaired RV function (TAPSE $\leq 17 \mathrm{~mm}$ ) & $8(72.7)$ \\
\hline Systolic PAP (mmHg) & $48.0(36.0,55.0)$ \\
\hline Systolic PAP $\geq 55 \mathrm{mmHg}$ & $3(27.3)$ \\
\hline$\geq$ Moderate TR & 7 (63.6) \\
\hline \multicolumn{2}{|l|}{ Computed tomography parameters } \\
\hline $\mathrm{CC}$ diameter $(\mathrm{mm})$ & $38.9(33.7,42.8)$ \\
\hline AP diameter $(\mathrm{mm})$ & $34.7(31.7,36.3)$ \\
\hline $\operatorname{Dmean}^{\mathrm{a}}(\mathrm{mm})$ & $38.1(32.4,39.4)$ \\
\hline Mitral annulus perimeter $(\mathrm{mm})$ & $127.0(118.5,131.9)$ \\
\hline Mitral annulus area $\left(\mathrm{cm}^{2}\right)$ & $12.1(10.5,12.9)$ \\
\hline CTA length (mm) & $91.7(82.5,99.1)$ \\
\hline Aorto-mitral angulation $\left({ }^{\circ}\right)$ & $134.3(118.5,138.5)$ \\
\hline Any MAC & $8(72.7)$ \\
\hline Severe circumferential MAC & $2(18.2)$ \\
\hline
\end{tabular}

Computed tomography parameters are presented as end-systolic measurements

$A P$ anterior-posterior, $C C$ intercommissural, $C T A$ center-to-apex, Dmean mean mitral annulus diameter, DMR degenerative mitral regurgitation, EROA effective regurgitant orifice area, FMR functional mitral regurgitation, $L V E D D$ left ventricular end-diastolic diameter, $L V E D V$ left ventricular end-diastolic volume, $L V E F$ left ventricular ejection fraction, $M A C$ mitral annulus calcification, $M R$ mitral regurgitation, $P A P$ pulmonary artery pressure, Pmean mean transvalvular gradient, $R V$ right ventricular, $S V I$ stroke volume index, TAPSE tricuspid annular plane systolic excursion, $T R$ tricuspid regurgitation

${ }^{\mathrm{a}}$ Dmean $=(\mathrm{IC}$ diameter $+\mathrm{AP}$ diameter $) / 2$ 
pulmonary artery pressure (sPAP) of $48.0 \mathrm{mmHg}$ (36.0, 55.0). Moderate or severe tricuspid regurgitation (TR) was assessed in $63.6 \%(N=7)$ of the study population.

Mitral annulus intercommissural (CC) diameters of all treated patients, as assessed by pre-procedural MSCT, ranged from $30.0 \mathrm{~mm}$ to $47.6 \mathrm{~mm}$ (measured in end-systole) with a median of $38.9 \mathrm{~mm}(33.7,42.8)$. Aorto-mitral angulation was $134^{\circ}(119,139)$. Any MAC was present in $72.7 \%$ $(N=8)$ of all patients. In two patients, severe circumferential MAC was identified.

\section{Procedural and in-hospital outcome}

Procedural parameters are given in Supplementary Table 2. Seven patients were treated with the Tendyne ${ }^{\mathrm{TM}}$ valve and four patients underwent Tiara ${ }^{\mathrm{TM}}$ implantation. All procedures $(N=11)$ were conducted via transapical access with transesophageal echocardiography guidance under general anesthesia. Technical success was achieved in all procedures (100.0\%) and all patients were extubated immediately after the procedure. Relevant LVOT obstruction was detected neither echocardiographically nor invasively. No patient required mechanical circulatory support during the procedure and there were no cases of conversion to open-heart surgery. Invasively measured cardiac index, assessed by Swan Ganz catheterization with thermodilution, increased from $1.71 \mathrm{~L} / \mathrm{min} / \mathrm{m}^{2}(1.55,1.98)$ before TMVR to $1.85 \mathrm{~L} / \mathrm{min}(1.71,2.38)(p=0.06)$. There was no procedural or in-hospital mortality ( $0.0 \%)$. After the procedure, all patients were prescribed lifelong oral anticoagulation with a vitamin K antagonist (INR 2.5-3.5) (with or without single anti-platelet therapy).

\section{Echocardiographic outcomes at discharge}

Detailed echocardiographic findings at discharge are given in Table 3. Mean transprosthetic gradients were low with a median of $3.0 \mathrm{mmHg}(3.0,5.0)$. One patient $(9.1 \%)$ was diagnosed with mild paravalvular leakage (PVL), three patients (27.3\%) had trace valvular MR and in 7 patients (63.6\%) no residual MR was detected. SVI after TMVR increased from $31.2 \mathrm{~mL} / \mathrm{m}^{2}(21.2,38.6)$ to $36.9 \mathrm{~mL} / \mathrm{m}^{2}$ $(32.0,44.1)(p=0.003)$ (Fig. 1a). Moreover, echocardiography after TMVR revealed a significant reduction in left ventricular end-diastolic volumes (LVEDV) (median $114.6 \mathrm{~mL}$ to $86.5 \mathrm{~mL}, p=0.03$ ) (Fig. 1b) and sPAP (median $48 \mathrm{mmHg}$

Table 3 MVARC 30-day and echocardiographic outcomes at discharge

\begin{tabular}{|c|c|}
\hline \multicolumn{2}{|l|}{ Echocardiographic outcomes } \\
\hline Pmean $(\mathrm{mmHg})$ & $3.0(3.0,5.0)$ \\
\hline$>$ trace $\mathrm{PVL}$ & $1(9.1)$ \\
\hline Mean LVOT gradient $(\mathrm{mmHg})$ & $1.8(1.7,3.6)$ \\
\hline Peak LVOT gradient $(\mathrm{mmHg})$ & $3.6(3.3,6.4)$ \\
\hline LVEDV (mL) & $86.5(79.1,125.5)$ \\
\hline $\operatorname{LVEF}(\%)$ & $39.0(35.0,50.0)$ \\
\hline $\mathrm{SVI}\left(\mathrm{mL} / \mathrm{m}^{2}\right)$ & $36.9(32.0,44.1)$ \\
\hline TAPSE & $15(12.3,16.0)$ \\
\hline Systolic PAP (mmHg) & $38.0(35.0,44.0)$ \\
\hline$\geq$ Moderate TR & $5(45.5)$ \\
\hline MVARC 30-day outcomes & $n=11$ \\
\hline 30-Day mortality & $0(0.0)$ \\
\hline Valve embolization/migration & $0(0.0)$ \\
\hline Valve thrombosis & $0(0.0)$ \\
\hline MV surgery/reintervention & $0(0.0)$ \\
\hline Myocardial infarction & $0(0.0)$ \\
\hline Disabling stroke & $0(0.0)$ \\
\hline Access-site complications & $2(18.2)$ \\
\hline Low cardiac output/inotrope therapy & $1(9.1)$ \\
\hline Major/life-threatening bleeding & $2(18.2)$ \\
\hline Renal failure ( $\geq$ AKIN 2) & $1(9.1)$ \\
\hline New-onset atrial fibrillation & $1(9.1)$ \\
\hline
\end{tabular}

$A K I N$ Acute Kidney Injury Network, $H F$ heart failure, $L V E D V$ left ventricular end-diastolic volume, $L V E F$ left ventricular ejection fraction, $L V O T$ left ventricular outflow tract, $M V$ mitral valve, $P A P$ pulmonary artery pressure, Pmean mean transvalvular gradient, $P V L$ paravalvular leakage, SVI stroke volume index, TAPSE tricuspid annular plane systolic excursion, $T R$ tricuspid regurgitation 
Fig. 1 Echocardiographic changes before and after TMVR. a Stroke volume index (SVI) $\left(\mathrm{mL} / \mathrm{m}^{2}\right)$. b Left ventricular end-diastolic volume (LVEDV). LVEDV left ventricular end-diastolic volume, $S V I$ stroke volume index, TMVR transcatheter mitral valve replacement
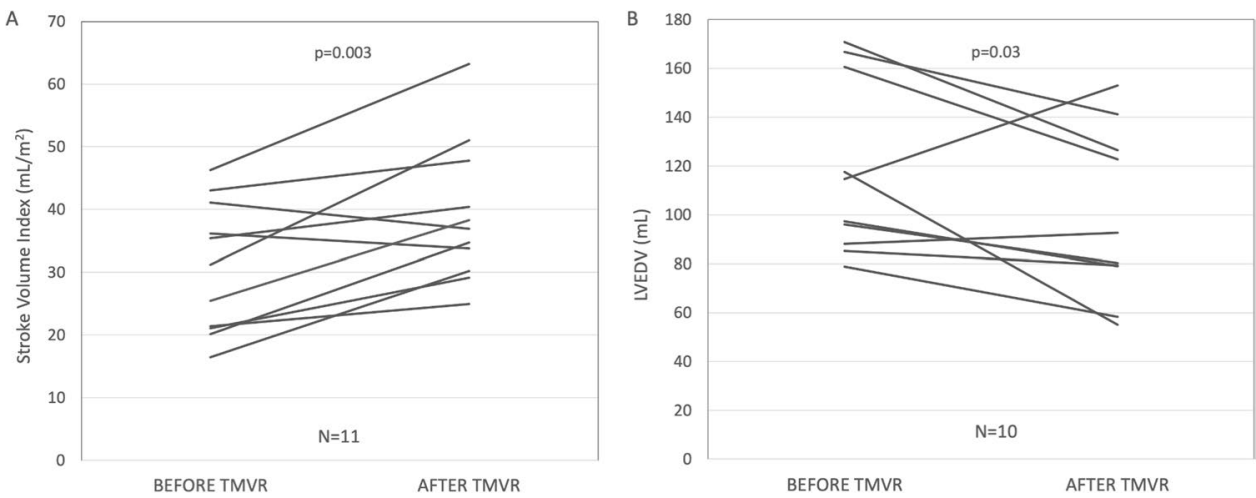

to $38 \mathrm{mmHg}, p=0.008)$. No significant post-procedural changes were found for LVEF or RV function.

\section{MVARC 30-day outcomes}

Detailed MVARC 30 day outcomes 30 days after TMVR are also displayed in Table 3. After 30 days all-cause mortality was still $0.0 \%$. Severe complications such as valve embolization or migration, valve thrombosis, mitral valve reintervention, myocardial infarction or disabling stroke did not occur within 30 days after TMVR. One patient required intermittent inotrope therapy due to temporary post-procedural low cardiac output. There were two cases of MVARC major bleeding due to access-site complications: one patient developed haemothorax with need for intermittent thoracic drainage installation. Another patient experienced secondary haemorrhage from the femoral puncture site. Acute kidney failure $\geq$ AKIN 2 occurred in one patient requiring postprocedural haemodialysis.

\section{Late echocardiographic and clinical outcomes after TMVR}

The severity of MR before TMVR and 30 days, 3 and 6 months after TMVR is demonstrated in Fig. 2. There were no cases of more than trace valvular MR. Figure 3 depicts the distribution of NYHA classes before TMVR and throughout all follow-up visits indicating functional improvement with a reduction to NYHA classes I or II in the majority of patients. After 79 and 97 days, two patients died after refractory resuscitation due to conduction disturbances in the first and due to an unknown cause in the second case resulting in all-cause mortality rates of $10.0 \%$ after 3 months and $22.2 \%$ after 6 months. All-cause mortality rate after 1 year was $33.3 \%$, as another patient expired after diagnosis of valve thrombosis following thrombolysis-related cerebral haemorrhage after 281 days. Mortality rates 30 days, 3 months, 6 months and 1 year after TMVR are summarized in Table 4. Longer term follow-up data of more than 2 years can be reported for 3 patients (follow-up:

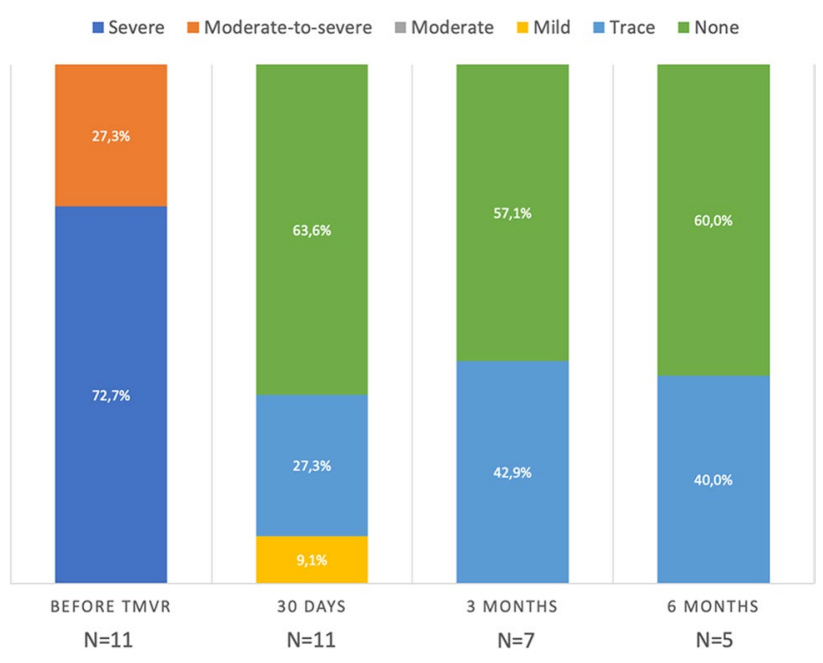

Fig. 2 Mitral regurgitation before TMVR and at follow-up. TMVR transcatheter mitral valve replacement

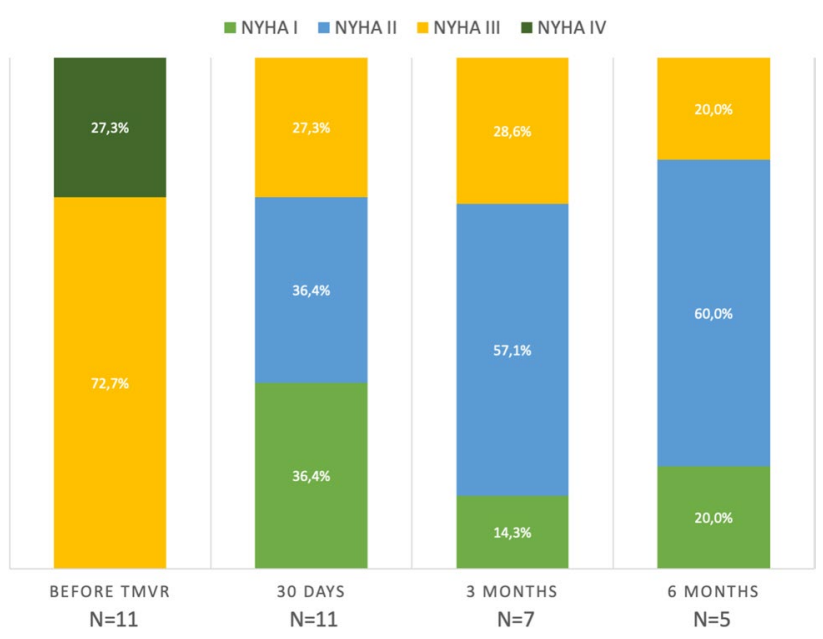

Fig. 3 NYHA class distribution before TMVR and at follow-up. NYHA New York heart association, TMVR transcatheter mitral valve replacement 
Table 4 Mortality rates 30 days, 3 months, 6 months and 1 year after TMVR

\begin{tabular}{lc}
\hline Follow-up & Mortality rates \\
\hline 30 days $(N=11)$ & $0.0 \%$ \\
3 months $(N=10)$ & $10.0 \%$ \\
6 months $(N=9)$ & $22.2 \%$ \\
1 year $(N=9)$ & $33.3 \%$ \\
\hline
\end{tabular}

$3.7,2.8$ and 2.3 years) treated in 2016 and 2017, all presenting with no or trace MR, mean transvalvular gradient 3-4 mmHg and NYHA stage II or III.

\section{Discussion}

This study reports one of the first series of TMVR implanted outside trial protocol-related restrictions. Patients were treated with the Tendyne ${ }^{\mathrm{TM}}$ valve (Fig. 4) or the Tiara ${ }^{\mathrm{TM}}$ valve (Fig. 5) either under a CU program or as CE implants, including the world's first CE implants with the Tendyne ${ }^{\mathrm{TM}}$
TMVR system. Our results suggest feasibility of TMVR in patients that were excluded from ongoing TMVR trials with a low procedural event rate for implanted TMVR devices, functional improvement according to NYHA class and elimination of MR in the majority of patients.

Recently published results of early feasibility trials with dedicated TMVR devices have impressively demonstrated the potential of TMVR as a true alternative for patients ineligible for established mitral valve therapies. Data from the early feasibility study of the Tendyne mitral valve system demonstrated safety of device implantation with the Tendyne $^{\mathrm{TM}}$ valve as well as functional improvement and effective reduction of MR [4]. The authors reported technical success in 96\%, 30-day and 1-year mortality rates of $6.0 \%$ and $26.0 \%$, respectively. In survivors, $88.5 \%$ experienced persisting symptom relief after 1 year. According to echocardiography at follow-up, LVEDV and SPAP decreased 1 year after TMVR [4]. Recently, the 2-year results were presented by Muller et al. with an all-cause mortality rate of $39.0 \%$ and no or trivial MR in $93.2 \%$ at 2 years after TMVR with the Tendyne ${ }^{\mathrm{TM}}$ valve [18].

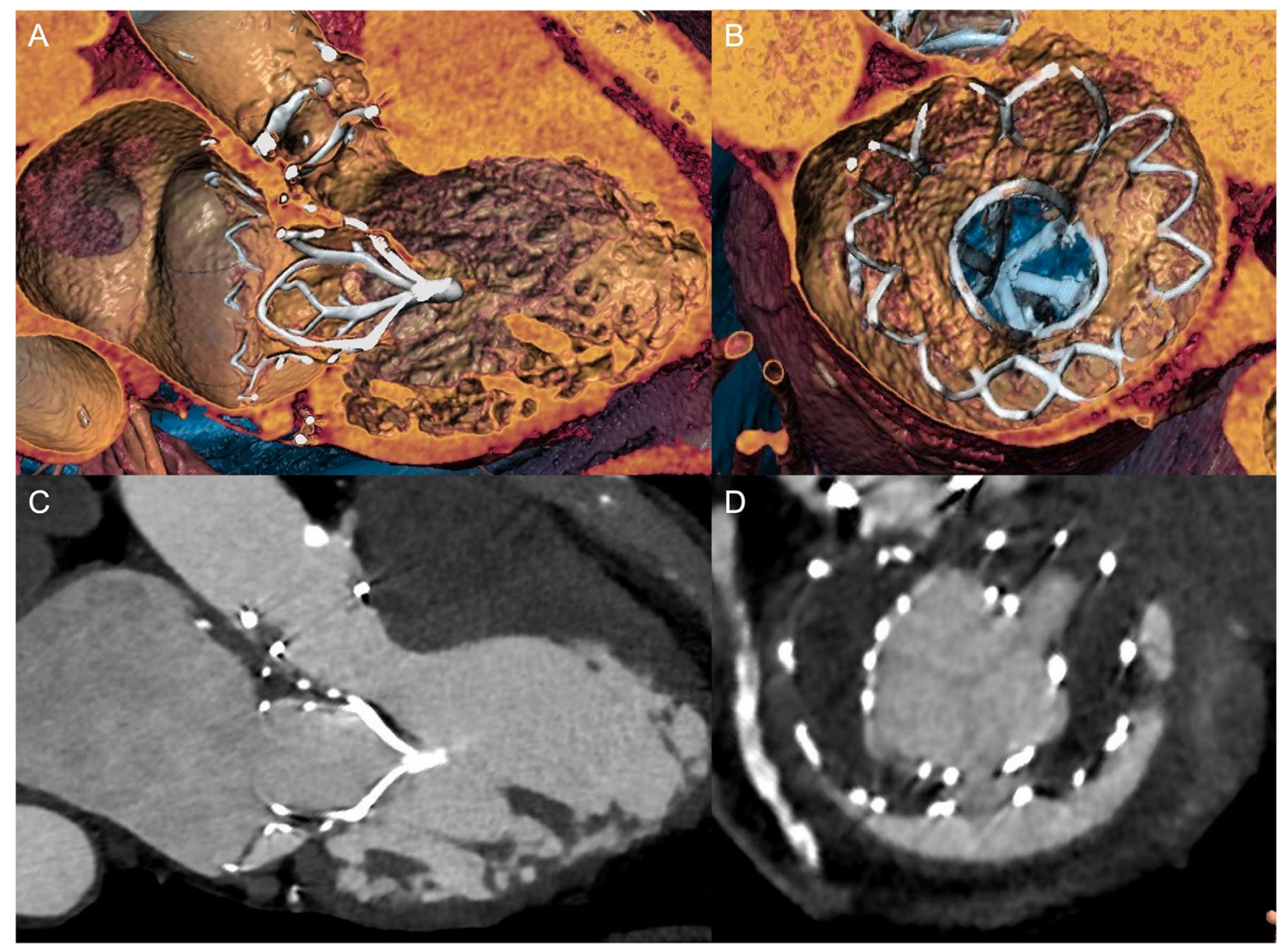

Fig. 4 Tendyne ${ }^{\mathrm{TM}}$ (Abbott Vascular) and prior AVR. (3Mensio Structural Heart, V10.0, Pie Medical Imaging, Maastricht, Netherlands). a $3 \mathrm{D}$ reconstruction of three-chamber view. b $3 \mathrm{D}$ en face view ("sur- geon's view") of the device. c 2D three-chamber view. d 2D en face view of the device 


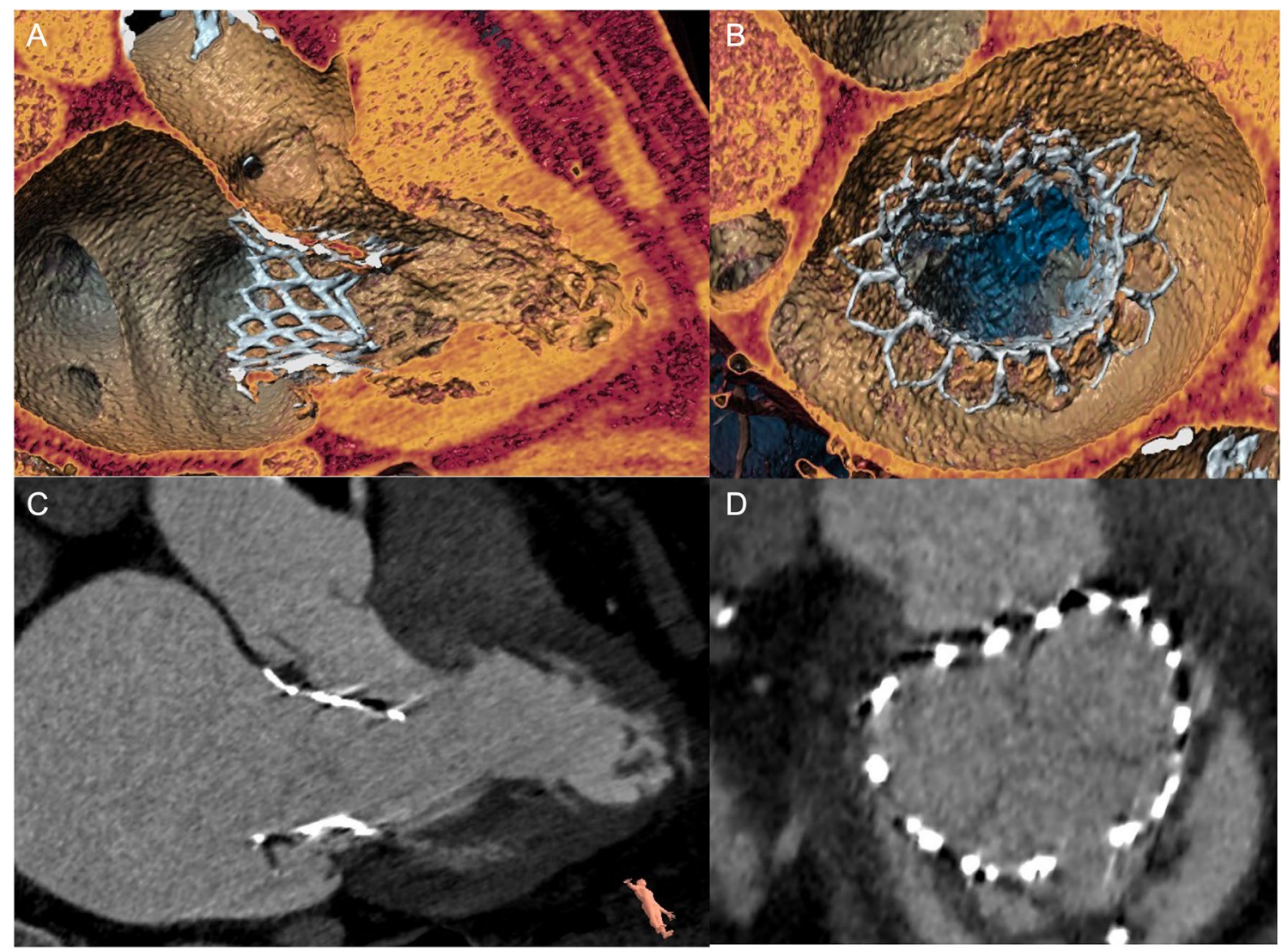

Fig. 5 Tiara ${ }^{\mathrm{TM}}$ (Neovasc Inc.). (3Mensio Structural Heart, V10.0, Pie Medical Imaging, Maastricht, Netherlands). a 3D reconstruction of threechamber view. b 3D en face view ("surgeon's view") of the device. c 2D three-chamber view. $\mathbf{d}$ 2D en face view of the device

The latest results of the early feasibility study of the Neovasc Tiara ${ }^{\mathrm{TM}}$ mitral transcatheter heart valve system (TIARA-I) and the Tiara ${ }^{\mathrm{TM}}$ Transcatheter Mitral Valve Replacement Study (TIARA-II) have recently been reported with high technical success, $0.0 \%$ procedural mortality and device-related mortality of $7.3 \%$ after 30 days [19]. Longterm results of these studies have not been published so far. However, resolution of MR was also observed in the majority of patients treated with the Tiara ${ }^{\mathrm{TM}}$ valve suggesting comparable findings [19].

Extensive exclusion criteria for ongoing early feasibility TMVR trials have led to high screening failure rates due to anatomical or clinical reasons $[5-7,20]$. While anatomical restrictions, such as risk of LVOT obstruction, annular dimensions and ventricular restraints largely depend on valve size and ventricular profile and, therefore, may at least partly be attenuated, clinical reasons for TMVR ineligibility mostly represent cautiousness of device manufacturers and reservations to perform TMVR in clinical conditions associated with adverse outcome [21-23]. Niikura et al. describe excessive frailty, severe TR and prior aortic valve replacement as common clinical reasons for TMVR exclusion [7]. Severe pulmonary hypertension, impaired LVEF and severe MAC represent further frequent factors associated with patient denial for TMVR [5, 6, 24].

The results of the herein presented real-world experience of patients with severe MR treated with two dedicated TMVR devices outside of company-funded feasibility trials demonstrate favourable echocardiographic and functional outcomes with both implanted devices. The study population is characterized by high prevalence of pulmonary hypertension, severe TR and impaired RV function, chronic kidney injury and high rates of prior surgical or interventional aortic valve replacement. Moreover, this cohort includes patients with severe circumferential MAC. Representing major study exclusion criteria these factors would have precluded those patients from interventional MR therapy via TMVR resulting in medical therapy only, which has previously shown to yield high mortality rates after TMVR screening failure [7, 8]. Similar to the above-discussed trials with the Tendyne ${ }^{\mathrm{TM}}$ and Tiara $^{\mathrm{TM}}$ valves, etiology of MR in the present study cohort was FMR $[18,19]$. Our study demonstrates feasibility of TMVR in patients with the above conditions, with technical success in $100.0 \%$ and 30 -day mortality of $0.0 \%$. After TMVR, echocardiographic assessment showed an increase 
in SVI and decreasing LV filling volumes correlating with effective symptom relief (NYHA class I/II) in almost all patients. Moreover, we can report complete resolution of MR in the majority of patients treated, persisting after 3 and 6 months. However, 1-year mortality rate was comparatively high, which may be explained by an overall reduced life expectancy due to coexisting comorbidities.

In accordance with our results, some studies have already demonstrated feasibility of TMVR in patients with special preconditions, such as MAC and prior aortic valve replacement $[10,11]$. Although these results are promising, results of ongoing trials like the MAC arm of the SUMMIT trial with the Tendyne $^{\mathrm{TM}}$ valve (NCT03433274) or the results of the TIARAI and -II (NCT02276547, NCT03039855) studies, will shed more light on this issue. Compared to other established interventional therapies targeting MR, the most important benefit achieved with TMVR may be the resolution of MR in almost all cases. Therefore, investigations addressing long-term effects of TMVR on LV remodelling and survival of patients are highly warranted. Recent studies have shown that residual MR and high mitral valve pressure gradients, especially in DMR patients, are associated with adverse outcome after endovascular edge-to-edge repair [25-27]. Prospectively, TMVR might constitute an alternative treatment option especially for patients with combined mitral valve disease (i.e. with small orifice, calcified leaflets, etc.), in whom MR elimination by edge-to-edge repair without high transvalvular gradients is unlikely.

\section{Limitations}

The presented study is limited by its study design and sample size. First, any drawn conclusions can only serve to generate hypotheses due to the retrospective study design. Second, the presented sample size is comparatively small. However, as TMVR represents a novel therapy, this singlecentre study represents the first report of patients treated successfully with TMVR outside of early feasibility trials.

\section{Conclusion}

In this real-world series of TMVR with two dedicated devices outside trial protocols, TMVR was performed successfully in all patients and complete elimination of MR was achieved in the majority of patients. Despite a high-risk profile, short-term mortality was low and patients experienced persisting functional improvement. These results suggest that TMVR has the potential to become an alternative treatment option for a broader subset of patients.

Funding Open Access funding enabled and organized by Projekt DEAL.. The authors did not receive specific funding for the conduct of this study.

\section{Compliance with ethical standards}

Conflict of interest SL received non-financial support from Edwards Lifesciences, outside the submitted work. MS reports non-financial support from Abbott Vascular, Biotronik, Boehringer Ingelheim, Boston Scientific, Edwards Lifesciences, Nicolai Medizintechnik, OrbusNeich Medical, personal fees from Abiomed, Amgen, AstraZeneca, Bayer Healthcare, Bristol-Myers Squibb, Boston Scientific, Medtronic and Philips and grants from Philips, outside the submitted work. US is a consultant for Abbott Vascular, received speaker honoraria and travel compensation from Abbott Vascular and received study grants from Abbott Vascular and Neovasc. DW reports personal fees from AstraZeneca, Bayer, Berlin-Chemie and Novartis, outside the submitted work. EL reports grants and personal fees from Abbott and personal fees from Edwards Lifesciences, during the conduct of the study. Outside the submitted work EL received personal fees from Abiomed, Astra Zeneca, Bayer, New Valve Technology and Novartis. LC received lecture fees from Abbott and Neovasc and is a proctor as well as advisory board member for Abbott and Neovasc during the conduct of the study. All other authors report no conflicts of interest.

Open Access This article is licensed under a Creative Commons Attribution 4.0 International License, which permits use, sharing, adaptation, distribution and reproduction in any medium or format, as long as you give appropriate credit to the original author(s) and the source, provide a link to the Creative Commons licence, and indicate if changes were made. The images or other third party material in this article are included in the article's Creative Commons licence, unless indicated otherwise in a credit line to the material. If material is not included in the article's Creative Commons licence and your intended use is not permitted by statutory regulation or exceeds the permitted use, you will need to obtain permission directly from the copyright holder. To view a copy of this licence, visit http://creativecommons.org/licenses/by/4.0/.

\section{References}

1. Regueiro A, Granada JF, Dagenais F, Rodés-Cabau J (2017) Transcatheter mitral valve replacement: insights from early clinical experience and future challenges. J Am Coll, Cardiol

2. del David V, Nunes FA, Jerome W et al (2019) Early experience with transcatheter mitral valve replacement: a systematic review. J Am Heart Assoc 8:e013332

3. Bapat V, Rajagopal V, Meduri C et al (2018) Early experience with new transcatheter mitral valve replacement. J Am Coll Cardiol 71(1):12-21. https://doi.org/10.1016/j.jacc.2017.10.061

4. Sorajja P, Moat N, Badhwar V et al (2019) Initial feasibility study of a new transcatheter mitral prosthesis: the first 100 patients. J Am Coll Cardiol 73(11):1250-1260. https://doi.org/10.1016/j. jacc.2018.12.066

5. Ludwig S, Ruebsamen N, Deuschl F et al (2020) Screening for transcatheter mitral valve replacement: a decision tree algorithm. EuroIntervention 16(3):251-258. https://doi.org/10.4244/ EIJ-D-19-01051

6. Coisne A, Pontana F, Tchétché D et al (2019) Transcatheter mitral valve replacement: factors associated with screening success and failure. EuroIntervention 15(11):983-989. https://doi.org/10.4244/ EIJ-D-19-00444

7. Niikura H, Gössl M, Kshettry V et al (2019) Causes and clinical outcomes of patients who are ineligible for transcatheter mitral valve replacement. JACC Cardiovasc Interv 12(2):196-204. https ://doi.org/10.1016/j.jcin.2018.10.042

8. Ludwig S, Koell B, Sedighian R, et al (2020) Characteristics and outcomes of patients ineligible for transcatheter mitral valve 
replacement (TMVR): implications for future device innovation. J Invasive Cardiol, accepted for publication.

9. Wallenborn J, Störk S, Herrmann S et al (2016) Prevalence of severe mitral regurgitation eligible for edge-to-edge mitral valve repair (MitraClip). Clin Res Cardiol 105:699-709

10. Cheung A, Schaefer U, Moss R et al (2018) Transcatheter mitral valve replacement in patients with previous aortic valve replacement. Circ Cardiovasc Interv 11:e006412

11. Sorajja P, Gössl M, Babaliaros V et al (2019) Novel transcatheter mitral valve prosthesis for patients with severe mitral annular calcification. J Am Coll Cardiol 74(11):1431-1440. https://doi. org/10.1016/j.jacc.2019.07.069

12. Baumgartner H, Falk V, Bax JJ et al (2017) 2017 ESC/EACTS guidelines for the management of valvular heart disease. Eur Heart J 38(36):2739-2791

13. Blanke P, Dvir D, Cheung A et al (2014) A simplified D-shaped model of the mitral annulus to facilitate CT-based sizing before transcatheter mitral valve implantation. J Cardiovasc Comput Tomogr 8(6):459-467

14. Quarto C, Davies S, Duncan A, et al (2016) Transcatheter mitral valve implantation 30 day outcome of first-in-man experience with an apically tethered device. In: Innovations: Technology and Techniques in Cardiothoracic and Vascular Surgery

15. Perpetua EM, Reisman M (2015) The Tendyne transcatheter mitral valve implantation system. EuroIntervention 11 Suppl W:W78-W79. https://doi.org/10.4244/EIJV11SWA23

16. Verheye S, Cheung A, Leon M, Banai S (2015) The Tiara transcatheter mitral valve implantation system. EuroIntervention. https://doi.org/10.4244/EIJV11SWA20

17. Cheung A, Webb J, Verheye $S$ et al (2014) Short-term results of transapical transcatheter mitral valve implantation for mitral regurgitation. J Am Coll Cardiol. https://doi.org/10.1016/j. jacc.2014.06.1208

18. Muller D, Sorajja P, Duncan A et al (2020) (2020) Two-year outcomes of Tendyne transcatheter mitral valve implantation to treat symptomatic. Severe Mitral Regurgitation, PCR e-course

19. Conradi L, Lubos E, Denti P, Cheung A (2020) Transcatheter Mitral Valve Implantation with Tiara: Transapical Results and Transseptal Design. PCR e-course 2020.
20. Meduri CU, Reardon MJ, Scott Lim D et al (2019) Novel multiphase assessment for predicting left ventricular outflow tract obstruction before transcatheter mitral valve replacement. JACC Cardiovasc Interv 12(23):2402-2412

21. Kitamura M, Kaneko H, Schlüter M et al (2019) Predictors of mortality in ischaemic versus non-ischaemic functional mitral regurgitation after successful transcatheter mitral valve repair using MitraClip: results from two high-volume centres. Clin Res Cardiol 108:264-272

22. Schueler R, Öztürk C, Sinning J-M et al (2017) Impact of baseline tricuspid regurgitation on long-term clinical outcomes and survival after interventional edge-to-edge repair for mitral regurgitation. Clin Res Cardiol 106:350-358

23. Zuern CS, Bauer A, Lubos E et al (2015) Influence of non-cardiac comorbidities on outcome after percutaneous mitral valve repair: results from the German transcatheter mitral valve interventions (TRAMI) registry. Clin Res Cardiol 104:1044-1053

24. Abbott Medical Deviced, Clinical Trial to Evaluate the Safety and Effectiveness of Using the Tendyne Mitral Valve System for the Treatment of Symptomatic Mitral Regurgitation (SUMMIT). https://clinicaltrials.gov/ct2/show/NCT03433274

25. Johannes P, Wenzhong Z, Reinhard S et al (2019) Elevated mitral valve pressure gradient is predictive of long-term outcome after percutaneous edge-to-edge mitral valve repair in patients with degenerative mitral regurgitation (MR). But Not in Functional MR, J Am Heart Assoc

26. Neuss M, Schau T, Isotani A et al (2017) Elevated mitral valve pressure gradient after MitraClip implantation deteriorates longterm outcome in patients with severe mitral regurgitation and severe hearrt failure. JACC Cardiovasc Interv 10(9):931-939. https://doi.org/10.1016/j.jcin.2016.12.280

27. Reichart D, Kalbacher D, Rübsamen N et al (2020) The impact of residual mitral regurgitation after MitraClip therapy in functional mitral regurgitation. Eur J Heart Fail. https://doi.org/10.1002/ ejhf.1774

\section{Affiliations}

\section{S. Ludwig ${ }^{1,2}$ - D. Kalbacher ${ }^{1,2} \cdot$ N. Schofer ${ }^{1} \cdot$ A. Schäfer ${ }^{3} \cdot$ B. Koell ${ }^{1} \cdot$ M. Seiffert ${ }^{1,2} \cdot$ J. Schirmer $^{3} \cdot$ U. Schäfer $^{4}$ D. Westermann ${ }^{1,2} \cdot$ H. Reichenspurner ${ }^{2,3} \cdot$ S. Blankenberg ${ }^{1,2} \cdot$ E. Lubos $^{1} \cdot$ L. Conradi $^{2,3}$}

S. Ludwig

se.ludwig@uke.de

1 Department of Cardiology, University Heart and Vascular Center Hamburg, Martinistrasse 52, 20246 Hamburg, Germany

2 Partner Site Hamburg/Kiel/Lübeck, German Centre for Cardiovascular Research (DZHK), Hamburg, Germany
3 Department of Cardiovascular Surgery, University Heart and Vascular Center Hamburg, Hamburg, Germany

4 Marienkrankenhaus Hamburg, Department of Cardiology, Angiology and Intensive Care, Hamburg, Germany 